\title{
La stratégie «Qualité» de la Confédération doit impliquer les partenaires!
}

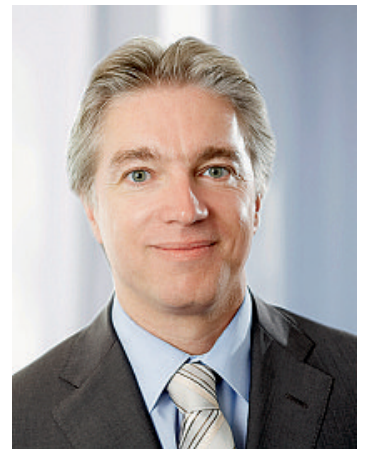

Enfin! La stratégie «Qualité» de la Confédération a enfin été dévoilée. Gardé secret pendant des mois, ce document a été l'objet d'innombrables spéculations. Désormais approuvé par le Conseil fédéral, il peut maintenant entrer en phase de concrétisation. Mais que renferment donc ces 121 pages? Restons brefs: cette stratégie correspond dans les grandes lignes à nos attentes car elle offre, si elle est judicieusement mise en œuvre, une chance de progresser dans le domaine de la qualité en Suisse.

La stratégie «Qualité» de la Confédération a pour noyau dur la création d'un organe national qui sera chargé de coordonner les démarches en matière de qualité dans les secteurs ambulatoire et hospitalier. On envisage donc de regrouper les activités en faveur de la qualité menées en Suisse et de les mettre en réseau. A cet effet, la Confédération a l'intention de distribuer clairement les rôles et de régler les responsabilités entre les acteurs. Sa stratégie prévoit neuf champs d'action propres à susciter des interventions ciblées en matière de qualité, par analogie à l'OMS. Ces champs sont, par exemple, la «direction et la responsabilité», les «informations en matière de qualité», les «incitatifs» ainsi que la création de ressources pour la mise en œuvre. Jusqu'ici, tout va bien.

\section{Nous demandons à l'OFSP d'impliquer dès le début le corps médical dans la mise en œuvre de la stratégie «Qualité» de la Confédération}

La FMH voit néanmoins deux points cruciaux dans la mise en œuvre de cette stratégie: premièrement, sa concrétisation doit absolument impliquer les différents acteurs de façon suffisante. Nous demandons à l'Office fédéral de la santé publique (OFSP) d'y inclure la FMH dès le début, conjointement avec les autres partenaires importants. En leur qualité de fournisseurs de prestations en contact direct avec les pa- tients, les médecins doivent participer à cette mise en œuvre afin qu'elle soit adéquate et utile. Nous sommes prêts à collaborer à cela de manière constructive.

\section{Des activités adéquates en matière de qualité coûtent de l'argent. Les 20 à 25 millions de francs prévus sont une somme modique et doivent être affectés en premier lieu à la mise en œuvre et à la coordination de la stratégie prévue}

Deuxièmement, la garantie et la mise à disposition du financement sont de première importance. Des activités adéquates et utiles en faveur de la qualité coûtent de l'argent. La stratégie prévue par la Confédération prévoit un budget de 20 à 25 millions de francs. Compte tenu des tâches du futur organe national chargé de la qualité, ce montant est modeste par rapport aux 60 milliards consacrés au système de santé. De ce fait, il est évident qu'un financement aussi modeste permettra au mieux d'élaborer une plate-forme axée avant tout sur la coordination et la stratégie. Mais ce qu'il conviendra absolument d'éviter, c'est la création d'un département supplémentaire de l'OFSP qui, isolé, tente d'appliquer une stratégie détachée de la pratique quotidienne, sans utilité quelconque pour personne.

Les projets sur la qualité des soins dirigés par la FMH (monitorage de la qualité, initiatives qualité et autres; cf. informations sur www.fmh.ch $\rightarrow$ Autres thèmes $\rightarrow$ Qualité) seront poursuivis jusqu'à la concrétisation de la stratégie de la Confédération et l'achèvement des travaux préparatoires. Compte tenu des intentions de l'OFSP, les projets de la FMH ont leur place dans le paysage helvétique sur la qualité des soins et ils pourront être intégrés dans la stratégie nationale indépendamment des structures qui lui seront données.

Dr Daniel Herren, MHA, Membre du Comité central de la FMH, Responsable du domaine DDQ 